\title{
Calculation of Pressure Pulsations in the Flow Part of the First Stage of an Axial Turbine in the Rotor-Stator Interaction Zone
}

\author{
S. F. Timushev ${ }^{1 *}$ and $A . V$. Kondratov ${ }^{1}$ \\ ${ }^{1}$ Rocket Engines Department, Moscow Aviation Institute (National Research University), 4 \\ Volokolamskoye HW, 125900, Moscow, Russian Federation
}

\begin{abstract}
A serious problem in the development of reusable liquidpropellant rocket engines (LRE) is the provision of a high resource and reliability of gas turbines of turbopump, which supply fuel to the combustion chamber. This problem can be solved by reducing the level of pressure pulsations in the interaction zone of the turbine rotor-stator and dynamic loads acting on the working and stator blades. In this regard, a useful tool is the method of numerical simulation of the unsteady turbulent flow of a compressible gas in the turbine flow path with the determination of the amplitude of pressure pulsations in the axial clearance between the stator and rotor blade cascades. The calculation model includes the Navier-Stokes equations and equation of energy. Density, thermal conductivity and diffusion coefficient are linearly dependent on temperature and concentration. Calculations were performed on different meshes, proving the mesh convergence of the method upon reaching the quasi-stationary regime. The calculation results show that the pressure pulsations vary greatly with the axial clearance, and the main frequency of the pressure pulsations in the spectrum is the blade passing frequency. The frequency of dynamic moment acting on the blade also coincides with the indicated frequency.
\end{abstract}

\section{Introduction}

The creation of new reusable transport rocket and space systems makes increased demands on the reliability and service life of the turbopump machines of LRE. At the same time, an increase in the efficiency and power density of LRE turbomachines brings to the fore the problems caused by unsteadiness of the flow: up to $70 \%$ of failures of modern liquidpropellant rocket engines are associated with damage to the structural elements of the fuel feed system due to the high level of vibration of the turbopump units, primarily turbines and centrifugal pumps. Non-stationary phenomena in turbomachines are expressed in a relatively high level of pressure pulsations and vibrations of the structure, non-stationary forces and moments acting on the rotor and shaft bearings, pressure fluctuations, which can, with an unfavourable combination of design and operating parameters, cause a resonant increase in

\footnotetext{
*Corresponding author: timushevSF@ mail.ru
} 
the amplitude of pressure fluctuations in the flow path of turbomachines, in the ducts with increasing vibration.

The unsteadiness is organically inherent in turbines, as well as in other bladed machines, since it is generated in the process of flow around rotating and stationary blade cascades.

At the same time, the vibration of pumps and other LRE units influences the vibration of the turbine. In unsteady flow and generation of pressure pulsations and vibration in a turbopump unit, an important role plays the interaction of vortex turbulent flows in the flow path, the presence of two-phase flow zones, resonant amplification of oscillations, and other factors. Uneven flow and pressure pulsations on the background of high pressure, temperature, oxidizing environment lead to an increase in stresses in the unit structure, the occurrence of fatigue damage, limiting its service life.

The use of methods and tools for the numerical analysis of oscillations recently comes to the fore in the design of turbomachines. Computational fluid dynamics (CFD) methods become the standard in turbine design and development. It is shown that necessary to account the influence of flow heterogeneity, separations and pressure pulsations on the decrease in efficiency when designing the last stages of steam turbines [1]. Simulation of unsteady flow in the first stage of the turbine for numerical analysis of noise generation mechanisms using a solver based on the RANS system of equations is performed and it is shown that the main source of noise is the hydrodynamic flow inhomogeneity during the interaction of blade rows, and temperature irregularity does not play a significant role [2]. The influence of small changes in the geometry of the cascade and the blade profile on the flow parameters [3] and on the aeroacoustic characteristics at the interaction of the flow inhomogeneity with the blade row was investigated to predict the phenomena of noise and aeroelasticity [4]. The problem of excitation of oscillations of the turbine casing structure under the influence of pressure pulsations in the form of standing and traveling wave oscillations is considered [5]. An unsteady flow, pressure pulsations and dynamic loads in a radial-axial turbine [6,7] and in an autonomous turbine with a partial supply [8] are investigated. In recent years, considerable attention has been paid to unsteady structural loads due to pressure pulsations arising in the rotor-stator zone, which can be responsible for the destruction of rotor blades $[9,10]$. CFDCAA methods are used to analyse the effect of the clearance between the blades of a lowpressure turbine on noise generated as a result of the rotor-stator interaction [11]. In this article, a study of pressure pulsations in the gap between the stator and rotor blades of the first stage of the turbine is carried out.

In the process of numerical modeling, mathematical models were used, included in the software package for computational fluid dynamics [12].

They describe the motion of a homogeneous medium at different velocities, taking into account the effects of compressibility, turbulence and heat transfer, including a model of heat transfer in a solid for the problem of conjugate heat transfer taking into account heat transfer between a gas and a solid, a k- $\varepsilon$ model of a turbulent fluid flow for modeling a gas flow at large and small Reynolds numbers for any Mach numbers.

When calculating conjugate heat and mass transfer and aerodynamics, the so-called reference values are set. The absolute values of the variables are determined through the reference and relative $(f)$ values as follows:

$$
f_{a b s}=f+f_{\text {ref }} .
$$

When determining the boundary and initial conditions, relative values are used. The system of finite-difference equations of computational fluid dynamics and heat transfer is solved by a non-stationary numerical method on a rectangular grid with local adaptation and subgrid resolution of complex geometry. In the computational domain, an initial grid with parallelepiped cells is defined. Geometry subdomains (boundary conditions) are highlighted, 
in which it is necessary to carry out the calculation on a denser mesh. In these subdomains, the initial computational grid is adapted, the cells of the initial grid are divided into 8 equal cells of the new adaptation level. Further, if necessary, the cells are divided again and so on, until the required accuracy is achieved. The cells of the initial mesh are called level 0 cells, the cells obtained by level 0 refinement are called level 1 cells, etc. The corresponding mesh is called the zero, first adaptation level mesh. The subgrid geometry resolution method is designed to approximate curvilinear boundaries on a rectangular mesh. The cells through which the border passes lose their original parallelepiped shape and turn into freeform polyhedrons. This approach makes it possible to perform calculations with a sufficient degree of accuracy, minimizing computational resources and processor time.

\section{Equations of hydrodynamics of a compressible medium and boundary conditions}

The model includes the Navier-Stokes equations, energy and turbulence equations. Density and thermal conductivity are linear with temperature.

- Navier-Stokes equations

$$
\begin{gathered}
\frac{\partial(\rho \mathbf{V})}{\partial t}+\nabla(\rho \mathbf{V} \otimes \mathbf{V})=-\nabla P+\nabla\left(\left(\mu+\mu_{t}\right)\left(\nabla \mathbf{V}+(\nabla \mathbf{V})^{T}\right)\right. \\
\frac{\partial \rho}{\partial t}+\nabla(\rho \mathbf{V})=0
\end{gathered}
$$

- $\quad$ Equation for total enthalpy

$$
\frac{\partial(\rho H)}{\partial t}+\nabla(\rho \mathbf{V H})=\frac{\partial P}{\partial t}+\nabla\left(\left(\lambda+\mu_{t}\right) \nabla T\right) .
$$

- Equation of state

$$
\rho=\frac{M}{R_{0}} \frac{P+P_{r e f}}{T+T_{r e f}}
$$

- $\quad$ Equations for $k$ and $\varepsilon$

$$
\begin{array}{r}
\frac{\partial(\rho k)}{\partial t}+\nabla(\rho \mathbf{V k})=\nabla\left(\left(\mu+\frac{\mu_{t}}{\sigma_{k}}\right) \nabla k\right)+G-\rho \varepsilon, \\
\frac{\partial(\rho \varepsilon)}{\partial t}+\nabla(\rho \mathbf{V} \varepsilon)=\nabla\left(\left(\mu+\frac{\mu_{t}}{\sigma_{\varepsilon}}\right) \nabla \varepsilon\right)+\frac{\varepsilon}{k}\left(C_{1} G-C_{2} \rho \varepsilon\right), \\
G=\mu_{t} \frac{\partial V_{i}}{\partial x_{j}}\left(\frac{\partial V_{i}}{\partial x_{j}}+\frac{\partial V_{j}}{\partial x_{i}}\right) .
\end{array}
$$

The values of the parameters of the $k-\varepsilon$ model are: $\sigma_{k}=1.0 ; \sigma_{\varepsilon}=1.3 ; C_{\mu}=0.09 ; C_{l}=1.44$; $C_{2}=1.92$.

Turbulent viscosity $\mu_{\mathrm{t}}$ is expressed in terms of $k-\varepsilon$ values as follows [13] 


$$
\mu_{t}=C_{\mu} \rho \frac{k^{2}}{\varepsilon} .
$$

\section{Geometry model}

The main parameters are shown in the table 1 .

Table 1. Main parameters.

\begin{tabular}{|c|c|c|}
\hline Parameter & Value & Unit \\
\hline Mass flow & 29,4 & $\mathrm{~kg} / \mathrm{s}$ \\
\hline Total inlet pressure & 329 & $\mathrm{~B}$ \\
\hline Inlet stagnation temperature & 1000 & $\mathrm{~K}$ \\
\hline
\end{tabular}

To carry out the research, a model of the first stage of the turbine was created, the blades of the rotor and stator parts were profiled (figure 1).

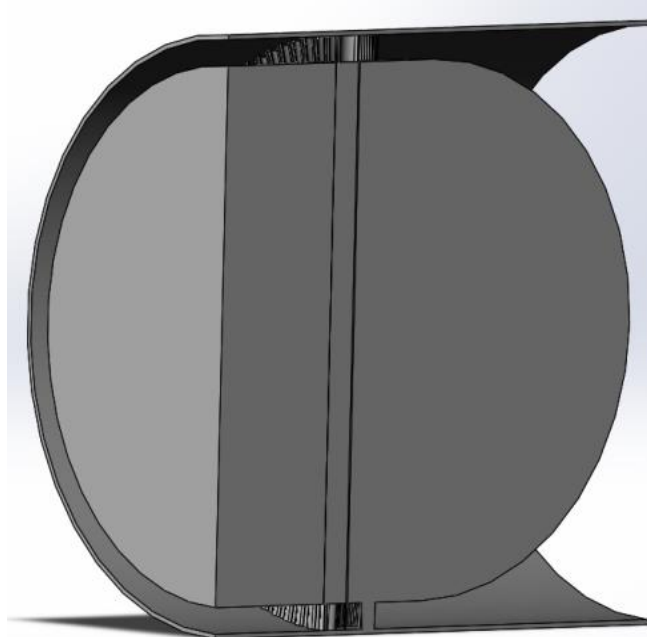

(a)

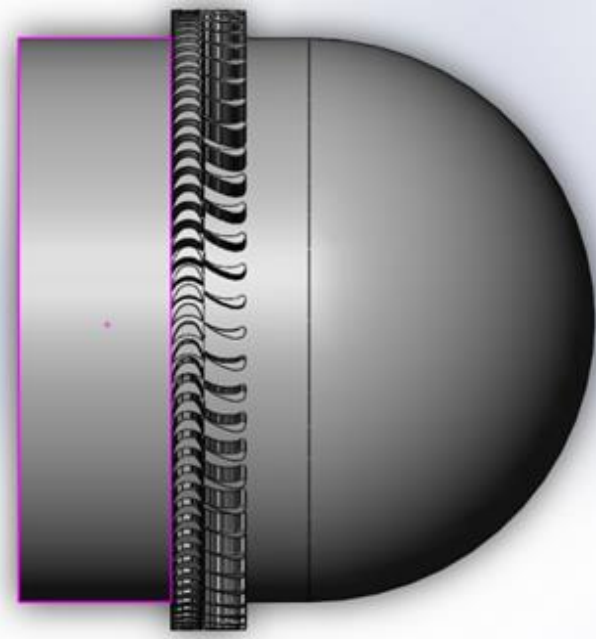

(b)

Fig. 1. Computational domain: meridional section (a); view of the cascade of nozzles and wheel (b).

The software package for gas dynamic calculations FlowVision was loaded with a solid three-dimensional model of the first stage of the turbine and the method of sliding surfaces is implemented when the model is divided into three subregions - the stator inlet with nozzle blades, the rotor and the stator outlet part (figure 2). 


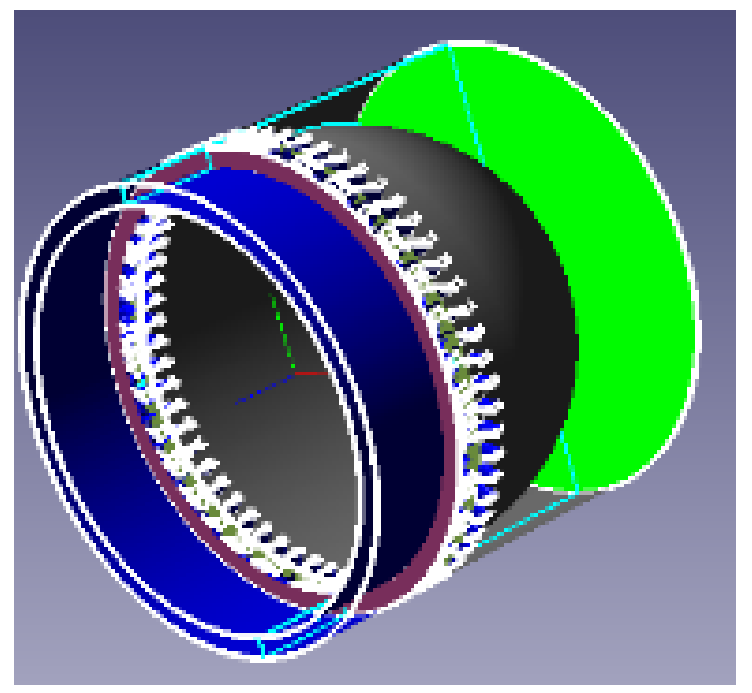

Fig. 2. Calculation area with boundary surfaces.

The boundary conditions are set as follows: the inlet is set to the value of the total pressure and the stagnation temperature of the gas indicated in the table. The output is set to the mass flow rate.

To study the pressure pulsations in the gap, in the Rotor-Stator zone, the points at which the static pressure is fixed are selected.

The study was carried out on several grids with different levels of adaptation to check the grid convergence. Two models were created with different axial gaps. For the first computation, the number of grid cells was 134013.

The maximum adaptation level is 2 . For calculations with a large axial gap, the number of calculation cells was 513282 (Three adaptation levels).

\section{Results}

The graph (figure 3) shows the gradual achievement of the convergence of the unsteady solution to a quasi-periodic oscillatory process with a constant amplitude, the turbine passes to a stationary mode of operation.

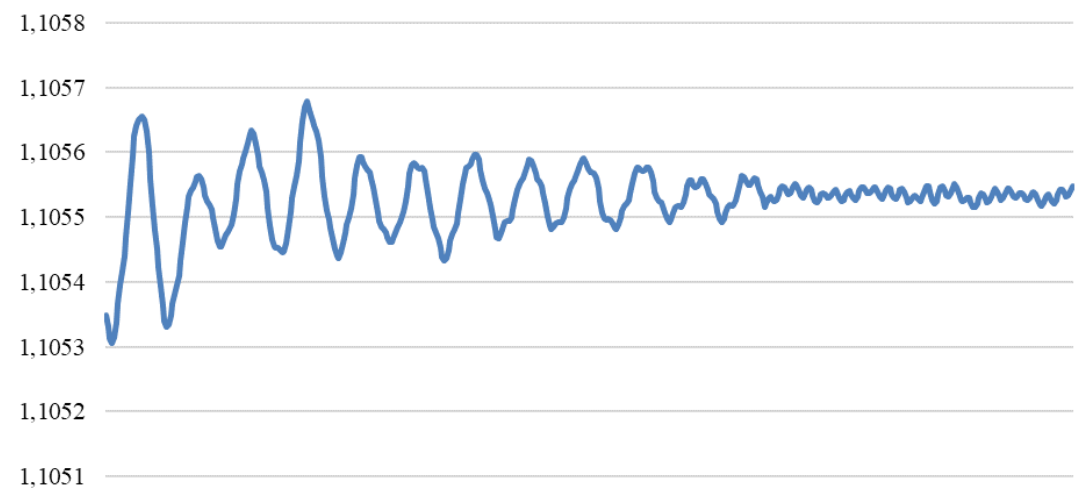

Fig. 3. Dependence of the pressure ratio at the inlet and outlet of the cascade. 
The amplitude of pressure pulsations in the gap was up to $2 \mathrm{MPa}$ at a gap of $6 \%$ of the chord.

With a gap of $12 \%$ of the profile chord, the calculation was carried out on grids with different levels of adaptation. The difference in the calculation results for the amplitude of pressure pulsations on the grids of the second and third adaptation levels is insignificant, therefore, the 2 nd adaptation level is sufficient to obtain results with acceptable accuracy.

Table 2. Comparison of the results.

\begin{tabular}{|c|c|c|}
\hline & Gap 6\% of the chord & Gap 12 \% of the chord \\
\hline Maximum level (MPa) & 33.62 & 32.75 \\
\hline Minimum level pressure (MPa) & 30.59 & 32.51 \\
\hline
\end{tabular}

Figure 4 shows the total signal and the spectrum of pressure pulsations in the rotor-stator zone at the axial gap of $6 \%$ of the blade chord. The spectrum is dominated by a discrete component at the Blade Passing Frequency (BPF) with an amplitude of 2.3 bar.

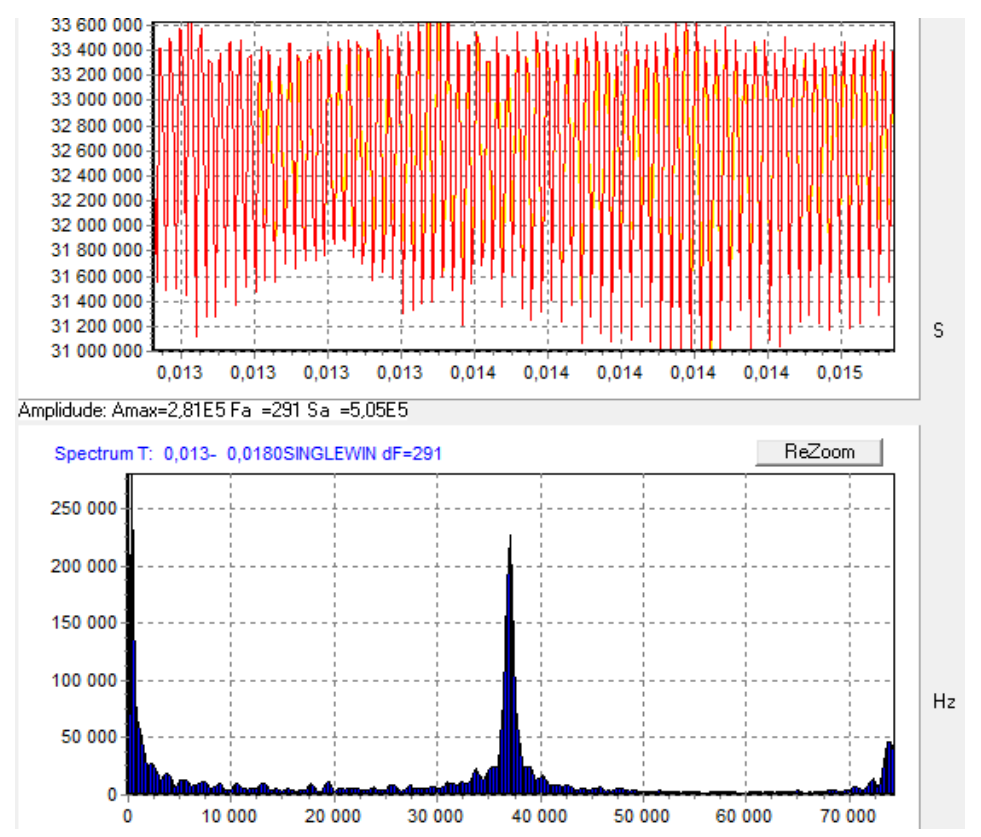

Fig. 4. Total signal and ripple spectrum (6\% chord gap).

\section{Conclusions}

The calculation of pressure pulsations in the rotor-stator interaction zone of the turbine first stage flow path at different axial gaps is carried out.

- For a gap size of $6 \%$ of the rotor blade chord, the maximum peak-to-peak amplitude is $3.15 \mathrm{MPa}$.

- For a gap size of $12 \%$ of the blade chord, the maximum peak-to-peak amplitude is $0.3 \mathrm{MPa}$.

The computation results show that the pressure pulsations strongly depend on the axial gap and the main frequency of the pressure pulsations coincides with BPF. 


\section{References}

1. G. G. Spenzer, Stopping Phenomena and Non-Stationary Processes in Wet Steam Stages of Low Pressure Cylinders of Steam Turbines. Scientific and technical bulletin of the St. Petersburg State Polytechnic University. 2 (219), 2015

2. D. Zante and E. Envia, Simulation of turbine tone noise generation using a turbomachinery aerodynamics solver 15th AIAA/CEAS Aeroacoustics Conference (30th AIAA Aeroacoustics Conference) (American Institute of Aeronautics and Astronautics Inc.), 2009

3. C. B. Lorence and Hall K. C. Sensitivity analysis of the aeroacoustic response of turbomachinery blade rows. 33rd Aerospace Sciences Meeting \& Exhibit (Reno: The American Institute of Aeronautics and Astronautics), 1995

4. R. Florea and Hall K. C. Sensitivity analysis of unsteady inviscid flow through turbomachinery cascades AIAA Journal 39 1047-56, 2001

5. Z. Y. Ma, Ran Y. C. and Zhang H. Z. Dynamic response analysis of powerhouse under turbine pressure pulsation with different distributions and application methods IOP Conference Series: Earth and Environmental Science vol 405 (Institute of Physics Publishing) p 12014, 2019

6. S. F. Timushev, D. V. Klimenko, V. P. Firsov and Antyukhov I. V. Numerical modeling of pressure pulsations and unsteady loads in a radial turbine of a turboexpander Proceedings of MAI 82, 2015

7. F. Hellström, Numerical computations of the unsteady flow in a radial turbine Technical Reports from Royal Institute of Technology KTH Mechanics Stockholm, Sweden, 2008

8. A. A. Aksenov, Ishaev R. O., Klimenko D. V. and Timushev S. F. Modeling of unsteady flow, pressure field and dynamic loads in an autonomous turbine. Abstracts of the Seventh All-Russian Conference "Computational Experiment in Aeroacoustics", 2018

9. E. Agnalt, Rotor Stator Interaction in Low-Specific Speed Francis Turbines. Thesis for the degree of Philosophiae Doctor. Norwegian University of Science and Technology Faculty of Engineering. Department of Energy and Process Engineering, 2019

10. E. Agnalt, Solemslie B. W., Storli P. T. S. and Dahlhaug O. G. The rotor-stator interaction onboard a low specific speed Francis turbine International Journal of Fluid Machinery and Systems 13 302-9, 2020

11. Z. Lei, Q. Weiyang, M. Zhongqiang and C. Pingping, The simulation of turbomachinery tone noise based on in-duct mode matching method 16th AIAA/CEAS Aeroacoustics Conference (31st AIAA Aeroacoustics Conference), 2010

12. Software package for gas and fluid flow simulation FlowVision. Version 2.5.0. Manual CAPVIDIA, 1999-2007 Leuven, Belgium

13. D. C. Wilcox, Turbulence modeling for CFD (Canada: DCW Industries, Inc.) 460 p., 1994 